\title{
Two-dimensional Pollutant Transport Simulations in Natural Streams with Horizontal Recirculation Zone
}

\author{
Inhwan Park ${ }^{1}$, Hoje Seong ${ }^{1}$, Hyung-jun $\mathrm{Kim}^{2}$ and Dong Sop Rhee ${ }^{2,1}$ \\ ${ }^{1}$ Korea Institute of Civil Engineering and Building Technology, Hydro Science and Engineering \\ Research Institute, Korea \\ ${ }^{2}$ Korea Institute of Civil Engineering and Building Technology, Multi Disaster Countermeasures \\ Organization, Korea
}

\begin{abstract}
In this study, flow and pollutant transport simulations were conducted in the tidal reach of the Han River where the horizontal recirculation zone induced by the tidal cycle causes the storage effect. The flow analysis results show that the flow direction was repeatedly changed due to the downstream water level and the horizontal recirculation zone was occurred at the point where the flow direction was changed. Thus, the pollutant cloud was advected back and forth by the periodically occurred reversal flow. The storage effects of the horizontal recirculation zone cause that the concentration-time curve shows oscillatory increasing patterns, and the retention time of pollutant cloud was also increased.
\end{abstract}

\section{Introduction}

In natural streams, the horizontal recirculation occurs due to geometrical complexities, channel confluent, and reversal flows etc. In aspect of channel geometries, abrupt channel expansion or contraction change flow patterns and cause the rotating flow at the corner of cross section [1]. In addition, sharply curved channel bend, which has relatively small the radius of curvature to channel width ratio, is one of the reason to develop the horizontal circulation zone [2]. The reversal flow can be created near the estuary, which has large tide amplitude, and the recirculation zone is also possibly to occur at the interface between the reversal flow and forward flow. Contaminants in rivers are trapped by the aforementioned recirculation zone, and estimates of the arrival time of peak concentration and the retention time of polluted water become difficult [3]. Therefore, the mixing properties in the recirculation zone are necessary to be investigated for prediction of pollutant mixing.

The Han River in South Korea inflows to the Yellow Sea, which has large tidal amplitude and the reversal flow in the period of rising tide. Thus, the horizontal recirculation zone induced by the reversal flow appears at the downstream of the Han River, and the recirculation zone transports back and forth according to the change of tidal constituents. In this tidal reach, [4-5] reported that the oscillatory breakthrough curves were obtained due to the effect of tidal conditions for the continuously spilled pollutants. The

\footnotetext{
${ }^{1}$ Corresponding author: $\underline{\text { dsrhee } @ \text { kict.re.kr }}$
} 
storage effect by the tidal current is known as the tidal trapping [6], and several researches [7-8] were published for analysis of the pollutant mixing, in which the retention time of pollutants was increased by the tidal cycle.

In this study, the two-dimensional (2D) flow and pollutant transport simulations were carried out in the Han River. From the flow analysis results, periodic change of flow patterns by the tidal cycle was reproduced using the depth-averaged hydrodynamic model. Furthermore, the accidentally spilled pollutant mixing was demonstrated by the unsteady flow analysis results. The 2D pollutant transport simulations were conducted by using the 2D particle dispersion model (2D PDM), which calculates pollutant mixing without inputting the dispersion coefficients, because the determination of the dispersion coefficients is difficult by the tidal currents [9].

\section{Model descriptions}

In this study, the $2 \mathrm{D}$ flow and pollutant mixing simulations were conducted using the depth-averaged flow and transport models. The 2D flow analysis model, named HDM-2D, was developed by [10] and the governing equation of the model is as below:

$$
\begin{aligned}
& \frac{\partial h}{\partial t}+h \frac{\partial \bar{u}_{i}}{\partial x_{i}}+\bar{u}_{i} \frac{\partial h}{\partial x_{i}}=0 \\
& \frac{\partial \bar{u}_{i}}{\partial t}+\bar{u}_{j} \frac{\partial \bar{u}_{i}}{\partial x_{j}}=-g \frac{\partial(H+h)}{\partial x_{i}}+\frac{\partial}{\partial x_{i}}\left(v \frac{\partial \bar{u}_{i}}{\partial x_{j}}\right)-\frac{g n^{2}}{h^{4 / 3}} \bar{u}_{i} \sqrt{\bar{u}_{j} \bar{u}_{j}}
\end{aligned}
$$

where $t=$ time; $h=$ water depth; $\bar{u}_{i}=(\bar{u}, \bar{v})=$ depth averaged velocity vector; $g=$ gravity acceleration; $H=$ bottom elevation; $v=$ kinematic viscosity; $n=$ Manning's roughness coefficient. The finite element method was adopted for the discretization of the governing equation of the HDM-2D.

The 2D pollutant mixing was simulated using the 2D PDM, which was developed by the step-by-step calculation procedure of shear advection and vertical diffusion based on the shear dispersion theory [11]. Furthermore, the 2D PDM model used the particle tracking technique to compute discrete computation procedures. The shear advection step is described in Eq. (3).

$$
x_{i}(z, t)=x_{i}(z, t)+u_{i}\left(x_{i}, z, t\right) \Delta t+R \sqrt{2 \varepsilon_{h} \Delta t}
$$

where $x_{i}=(x, y)=$ horizontal particle position; $u_{i}=(u, v)=$ vertical distributions of longitudinal and transverse velocities; $R=$ Random number generated by the Box-Muller transform; $\varepsilon_{h}=$ horizontal diffusion coefficient $=\beta h u^{*}(\beta=0.15-0.30) ; u^{*}=$ shear velocity. By the shear advection step, the depth-averaged pollutant column is transported by the shear flow, in which $u$ and $v$ were generated by the log profile ([12]) and linear profile ([13]), respectively. After the shear advection step, the stretched pollutant column by the shear flow is well mixed by the vertical mixing step as below:

$$
\begin{array}{ll}
x_{i}(z, t)=x_{i}\left(z+R \sqrt{\varepsilon_{V} \Delta t}, t\right) & \text { for } \alpha n_{p}\left(x_{i}, t\right) \\
x_{i}(z, t)=x_{i}(z+h / L, t) & \text { for }(1-\alpha) n_{p}\left(x_{i}, t\right)
\end{array}
$$


where $\alpha=\Delta t / t_{v m} ; t_{v m}=$ time for vertical mixing in the vertical direction; $n_{p}=$ the number of particles in a water column; $L=$ the number of vertical layer; $\varepsilon_{V}=$ vertical diffusion coefficient $=0.067 \mathrm{hu}^{*}$. In the vertical mixing step, advected pollutant particles by the shear flow were redistributed in a water column, and the pollutant particles were advected again by the shear flow using Eq. (3) in the next time step. From these computation steps, the pollutant mixing can be calculated without limitations of adopting the Fick's law, which assumes that the 2D mixing can be simulated only after the initial period [14].

\section{Tidal trapping by the horizontal recirculation zone}

In this study, 2D flow and pollutant mixing simulations were conducted in the tidal reach of the Han River, which describes in Figure 1. In this study area, several bridges were across the Han River for connecting the north and south area. Thus, this study area is vulnerable to be polluted by car accidents. Furthermore, the complex flow patterns induced by the tidal flow cause difficulty to predict behaviour pollutant cloud. The downstream water level (Hang-ju Bridge) is affected by the tidal currents of the Yellow Sea, and the water level changes with periodic patterns in the period of $24 \mathrm{hrs}$. The time series of the upstream flowrate (Cheongdam Bridge) and the downstream water level shown in Figure 2 clearly indicate the aforementioned flow characteristics in flood season of the study reach.

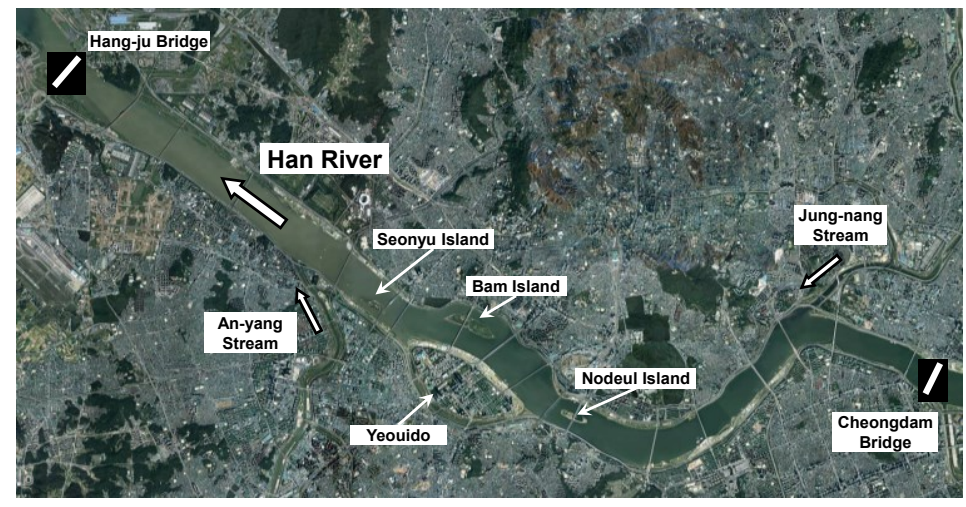

Fig. 1. Descriptions of the study reach in the Han River (ref., DAUM satellite map).

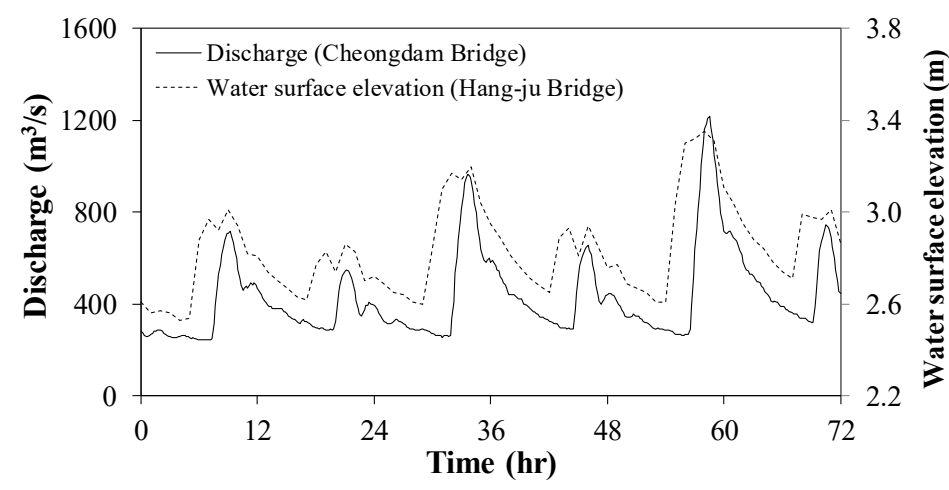

Fig. 2. Unsteady flow conditions in the flood season of the study reach 
Flow patterns by the periodic boundary conditions shown in Fig 2 were reproduced by using the HDM-2D. For the simulation using the HDM-2D, input parameters were determined following the study of [9], in which $v$ is $4 \mathrm{~m}^{2} / \mathrm{s}, n$ is 0.025 in the main channel, and $n=0.035$ in the sidewall. The computational mesh for simulation of flow mixing was depicted in Fig. 3, in which the total number of node is 4460 and the number of element is 4890. Fig. 4 shows velocity vectors, in which the flow direction was repeatedly changed due to the downstream water level. At $t=46 \mathrm{hr}$, downstream water level rises to the local maximum from the lowest level, and the reversal flow was reached to the upstream of the Nodeul Island. The water level begins to decrease again and the flow direction was changed to the forward direction. Thus, at $t=48 \mathrm{hr}$, the large horizontal recirculation zone was occurred at the border of backward and forward flows.

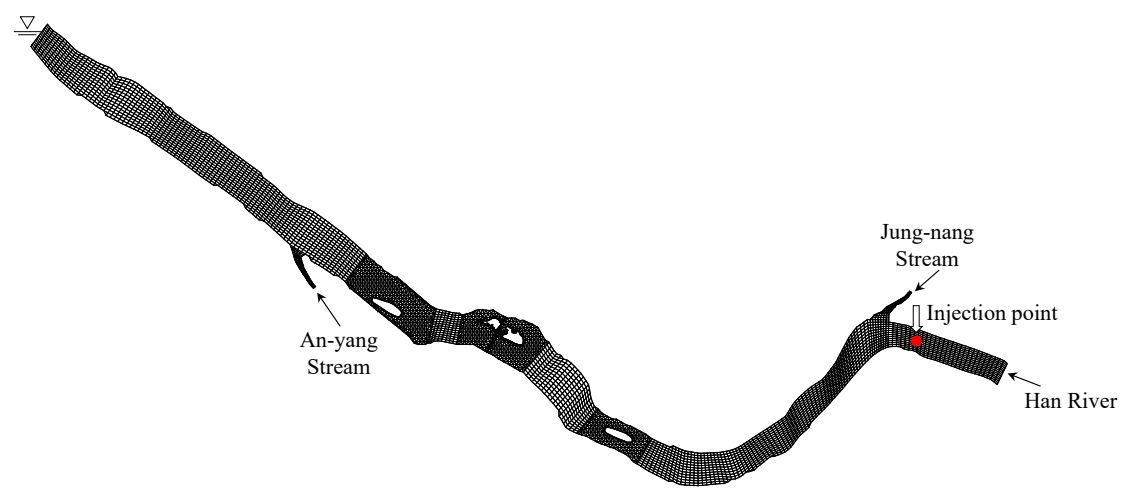

Fig. 3. Computational mesh for simulations of flow and pollutant mixing

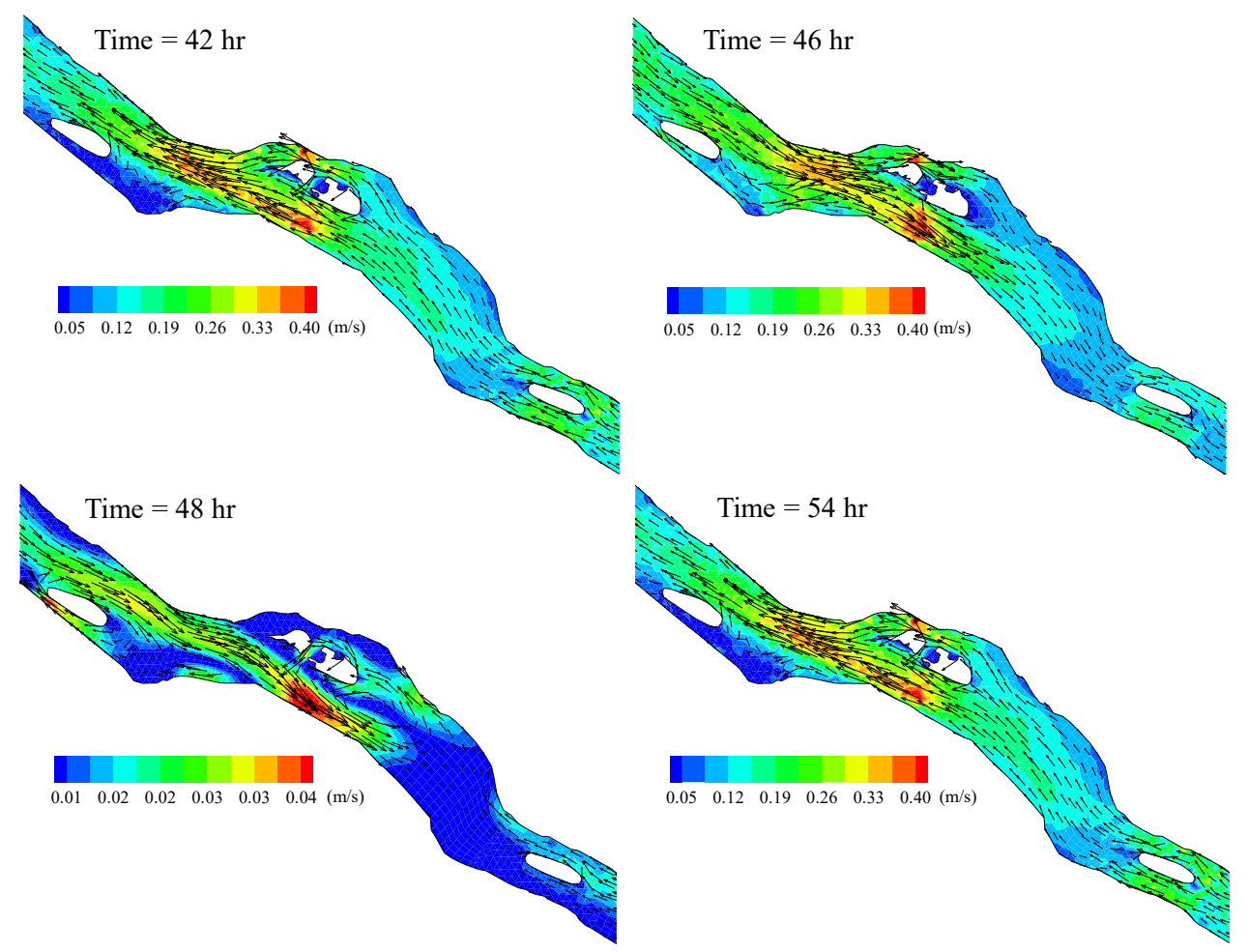

Fig. 4. Reversal flow occurrences in the study reach 
In the assumption of the water pollution accident, 10,000 particles with 1 ton of mass were instantaneously introduced at the center of channel when $t=0 \mathrm{hr}$ as shown in Fig. 3. Pollutant transport simulation was conducted using 2D PDM, and the concentration of particles was calculated using Eq. (5).

$$
C(x, \mathrm{y})=\frac{m}{h \Delta x \Delta y} n_{p}(x, \mathrm{y})
$$

where $C(x, y)=$ depth-averaged concentration; $\Delta x, \Delta y=$ grid size; $m=$ mass of a pollutant particle. Fig. 5 shows the simulation results of pollutant mixing in the flow conditions shown in Fig. 4. After $t=46 \mathrm{hr}$, flow direction is changed to the backward direction due to the increase of downstream water level. Thus, the pollutant cloud was advected to the upstream direction, and the pollutant particles were trapped by the horizontal recirculation zone occurred at the downstream of the Bam Island. After $t=54 \mathrm{hr}$, the pollutant cloud was transported back and forth according to the flow direction change.

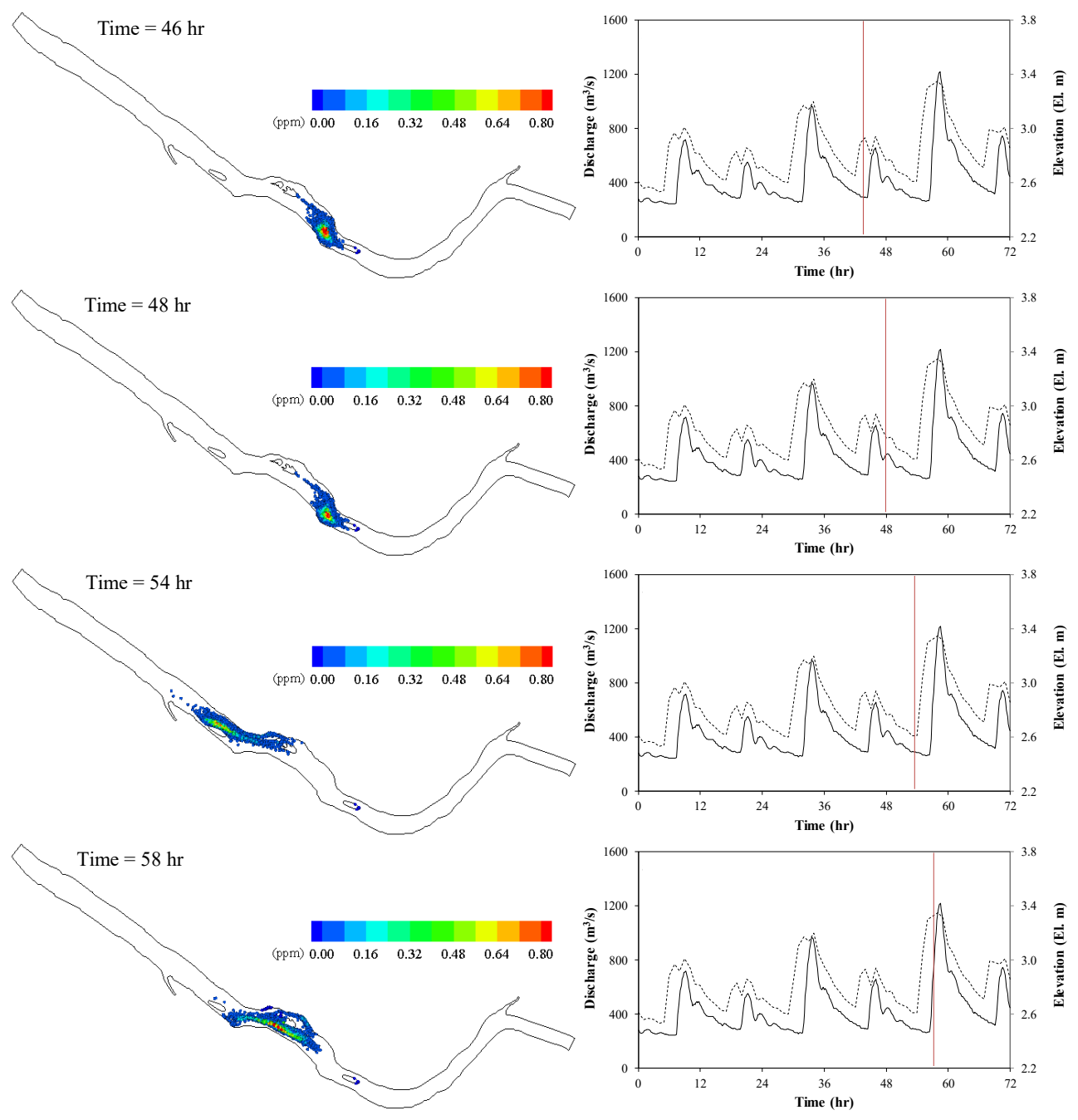

Fig. 5. Pollutant mixing in the horizontal recirculation zone 
From the simulation results shown in Fig. 5, the concentration-time curves at the Seonyu and Yeoui Islands, where the riverine parks are located, were compared in Fig. 6. On account of the periodic change of flow direction, the peak concentration was repeatedly occurred at the measuring points. In both measuring points, peak concentration was gradually increased by the superposition of pollutant particles in the horizontal recirculation zone. Especially, at the Seonyu Island, the concentration-time curve shows oscillatory increasing pattern, which also increases the retention time of polluted water. The peak concentration appears with $4 \mathrm{hrs}$ interval at the Seonyu Island and $10 \mathrm{hrs}$ interval at the Yeoui Island. Thus, the disaster prevention plan should be considered the tidal trapping induced by the reversal flow.

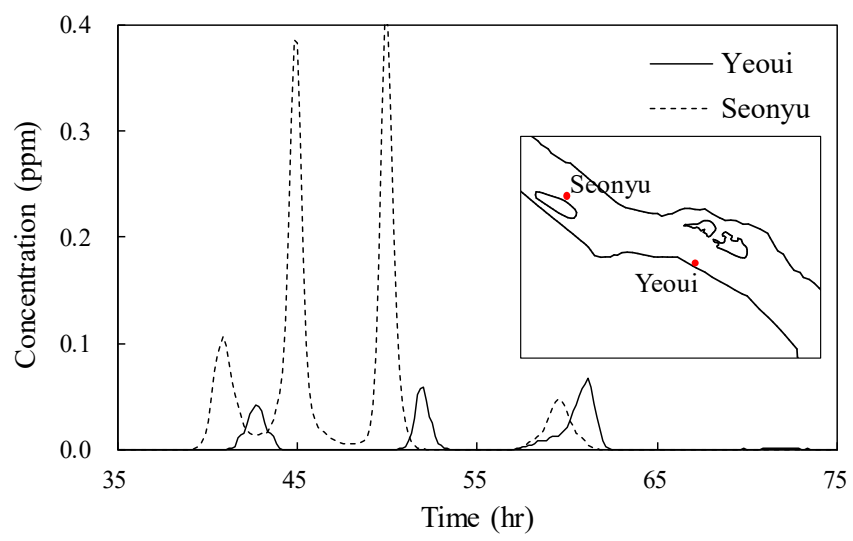

Fig. 6. Concentration-time curves near the riverine parks

\section{Conclusions}

In this study, the 2D flow and pollutant mixing simulations were conducted in the tidal reach of the Han River. The flow analysis results show that the flow direction was periodically changed due to the tidal cycle of the Yellow Sea. Thus, the reversal flow was reached to the upstream of the Nodeul Island, and the horizontal recirculation zone was occurred at the border of forward and backward flows. Thus, the polluant cloud was advected back and forth by the flow direction change, and the pollutant cloud was trapped in the horizontal recirculation zone. These mixing properties cause the oscillatory increasing concentration-time curve at the riverine park of the Seonyu Island, and the peak concentration was repeatedly occurred by the tidal currents.

\section{Acknowledgement}

This research was supported by a grant (17CTAP-C095650-03) from Infrastructure and Transportation Technology Promotion Program funded by Ministry of Land, Infrastructure and Transport of Korean government and a grant from the Strategic Research Project (Smart Flood Management) funded by the Korean Institute of Civil Engineering and Building Technology

\section{References}

1. I. Park, I. W. Seo, Kim, Y. D., C. G. Song, Environ. Earth Sci., 75, 2 (2016)

2. E. J. Hickin, Canadian J. Earth Sci., 15, 11 (1978) 
3. I. Park, C. G. Song, J. Hydroinform., 19, 6 (2017)

4. M. E. Lee, I. W. Seo, J. Hydro-Environ. Res., 1, 1 (2007)

5. M. E. Lee, I. W. Seo, KSCE J. Civil Eng., 14, 1 (2010)

6. H. B. Fischer, J. E. List, R. C. Y. Koh, J. Imberger, N. H. Brooks, Mixing in inland and coastal waters (Academic Press, 1979)

7. P. V. Ridd, E. Wolanski, Y. Mazda, Estuar. Coast. Shelf Sci., 31, 5 (1990)

8. F. L. Hellweger, A. F. Blumberg, P. Schlosser, D. T. Ho, T. Caplow, U. Lall, H. Li, Estuar., 27, 3 (2004)

9. L. J. MacVean, M. T. Stacey, Estuar. Coast, 34, 1 (2011)

10. I. W. Seo, Y. D. Kim, C. G. Song, KSCE J. Civil Eng., 19, 6 (2014)

11. I. Park, I. W. Seo, Adv. Water Resour., 111, 105-120 (2018)

12. I. L. Rozovskii, Flow of water in bends of open channels (Academy of science of Ukrainaian SSR, Russia, 1957)

13. A. J. Odgaard, J. Hydraul. Eng., 112, 12 (1986)

14. H. B. Fischer, Methods for predicting dispersion coefficients in natural streams with applications to lower reaches of the Green and Duwamish Rivers Washington (US Government Printing Office, Washington, 1968) 